\title{
2XMM J083026+524133: the most X-ray luminous cluster at redshift 1
}

\author{
G. Lamer, M. Hoeft, J. Kohnert, A. Schwope, and J. Storm
}

\author{
Astrophysikalisches Institut Potsdam, An der Sternwarte 16, 14482 Potsdam, Germany \\ e-mail: glamer@aip.de
}

Received 25 May 2008 / Accepted 23 June 2008

ABSTRACT

\begin{abstract}
Aims. In the distant universe X-ray luminous clusters of galaxies are rare objects. Large area surveys are therefore needed to probe the high luminosity end of the cluster population at redshifts $z \gtrsim 1$.

Methods. We correlated extended X-ray sources from the second XMM-Newton source catalogue (2XMM) with the SDSS in order to identify new clusters of galaxies. Distant cluster candidates in empty SDSS fields were imaged in the $r$ - and $z$-bands with the Large Binocular Telescope. We extracted the X-ray spectra of the cluster candidates and fitted thermal plasma models to the data.

Results. We determined the redshift $0.99 \pm 0.03$ for 2 XMM J083026.2+524133 from its X-ray spectrum. With a bolometric luminosity of $1.8 \times 10^{45} \mathrm{erg} \mathrm{s}^{-1}$ this is the most X-ray luminous cluster at redshifts $z \geq 1$. We measured a gas temperature of $8.2 \pm 0.9 \mathrm{keV}$ and estimate a cluster mass $M_{500}=5.6 \times 10^{14} M_{\odot}$. The optical imaging revealed a rich cluster of galaxies.
\end{abstract}

Key words. galaxies: clusters: individual: 2XMM J083026.2+524133 - X-rays: galaxies: clusters

\section{Introduction}

Clusters of galaxies play an important role as cosmological probes and as laboratories for the evolution of galaxies. There is observational evidence that the cosmological evolution of the cluster X-ray luminosity function makes very massive, X-ray luminous clusters rare objects at high redshifts (e.g. Henry et al. 1992; Rosati et al. 2002). At the same time, simulations show that the number counts of the most massive distant clusters depend strongly on the cosmological parameters, making them the most interesting cluster sample for cosmological studies (e.g. Borgani \& Guzzo 2001; Romer et al. 2001).

After the identification of the ROSAT surveys only a handful of clusters at redshifts beyond $z=1$ were known, since only few ROSAT observations were deep enough to reach the necessary flux limits. The identification of serendipitously detected $\mathrm{XMM}-$ Newton sources pushed the redshift limit for X-ray selected clusters beyond $z=1.4$, where two clusters with X-ray luminosities of several $10^{44} \mathrm{erg} \mathrm{s}^{-1}$ have been discovered (Mullis et al. 2005; Stanford et al. 2006). Clusters at redshifts $z \sim 1$ are now routinely identified from serendipitous XMM-Newton sources or in dedicated survey fields (e.g. Pierre et al. 2006; Finoguenov et al. 2007). However, the majority of these clusters have luminosities of only few times $10^{43} \mathrm{erg} \mathrm{s}^{-1}$ or less, placing them in the transition regime between clusters and groups of galaxies. In order to probe the high-luminosity part of the distant cluster population, large area surveys are needed to find high luminosity, distant clusters. To date the largest medium sensitivity $\mathrm{X}$-ray survey is the serendipitous XMM-Newton survey, which contains all sources found with the EPIC cameras in the public XMM-Newton observations.

In Sect. 2 of this paper we describe the selection of cluster candidates from XMM-Newton sources. The analysis of the X-ray data and optical observations of a new distant and very luminous cluster is described in Sects. 3 and 4 respectively. Section 5 concludes the paper with a brief discussion and outlook.

The cosmological parameters $\Omega_{\mathrm{M}}=0.3, \Omega_{\Lambda}=0.7$ and $H_{0}=$ $71 \mathrm{~km} \mathrm{~s}^{-1} \mathrm{Mpc}^{-1}$ have been used throughout this paper, unless mentioned otherwise. With these parameters the linear scale at $z=1.0$ is $7.9 \mathrm{kpc} /{ }^{\prime \prime}$.

\section{Cluster candidate selection}

We have started a programme to identify the extended source content of the second XMM-Newton catalogue (2XMM, Watson et al. 2008). $2 \mathrm{XMM}$ is based on a compilation of $\sim 3500 \mathrm{EPIC}$ observations performed before May 2007. It covers a sky area of 360 square degrees and comprises 192000 unique X-ray sources. The source detection software, based on the XMM-SAS version 7.1, was configured to discern point like and extended $\mathrm{X}$-ray sources and a $\beta$-model profile is fitted to the images of extended sources. Since the spurious detection rate among the 20 000 extended sources in 2XMM is relatively high, we have cleaned the sample of extended sources for obviously spurious detections by means of visual inspection of the X-ray images.

We have correlated the confirmed extended sources with the spectroscopic and photometric databases of the Sloan Digital Sky Survey (SDSS). The 2XMM survey area within the SDSS DR6 footprint is $\sim 120$ square degrees. The results on the 2XMM cluster candidates detected in the SDSS will be reported elsewhere. For a relatively small number of extended $\mathrm{X}$-ray sources no cluster or galaxy counterpart is visible in the SDSS images. These sources can be considered as candidates for distant clusters beyond redshifts of $\sim 0.8$. The cluster 2XMM J083026.2+524133 was the brightest X-ray source among these "empty field" candidates. 


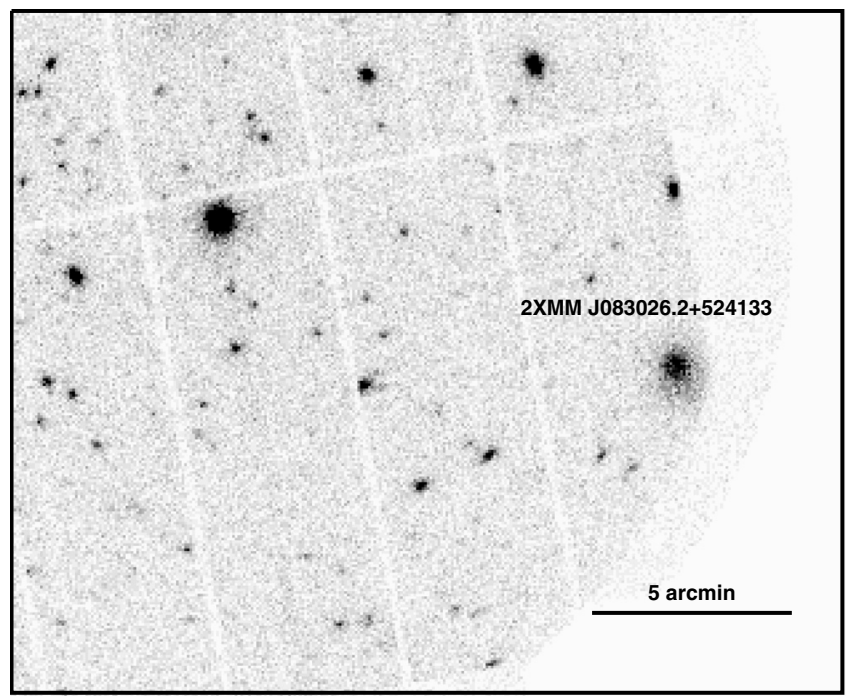

Fig. 1. EPIC MOS1 + PN image of observation 0092800201 in the $0.2-4.5 \mathrm{keV}$ energy band.

Table 1. 2XMM 0830: 2XMM catalogue source parameters.

\begin{tabular}{lll}
\hline \hline ObsID & 0092800101 & 0092800201 \\
\hline PN parameter & 13896 & 72133 \\
ONTIME [s] & 13.2 & 10.9 \\
OFFAX ["] & $0.148 \pm 0.009$ & $0.115 \pm 0.003$ \\
CR .2-12 keV $\left[\mathrm{s}^{-1}\right]$ & & \\
PN flux & & \\
$0.2-12 \mathrm{keV}\left[10^{-13} \mathrm{erg} / \mathrm{cm}^{2} \mathrm{~s}\right]$ & $4.79 \pm 0.99$ & $3.46 \pm 0.28$ \\
$0.5-2 \mathrm{keV}\left[10^{-13} \mathrm{erg} / \mathrm{cm}^{2} \mathrm{~s}\right]$ & $1.40 \pm 0.10$ & $1.11 \pm 0.03$ \\
COUNTS & $627 \pm 37$ & $3457 \pm 84$ \\
\hline
\end{tabular}

\section{X-ray data}

2XMM J083026.2+524133 has been serendipitously detected in XMM EPIC observations of the quasar APM 08279+5255 (Observation IDs 0092800101, 0092800201) and entered the 2XMM source catalogue as an extended X-ray source. The extended nature was clearly confirmed by our visual screening (see Fig. 1). Some catalogue parameters of the source, henceforth referred to as 2XMM 0830 are summarised in Table 1.

With more than 4000 detected source counts in the EPIC PN camera the source is bright enough to attempt a redshift determination based on its X-ray spectrum. We extracted the source counts from the merged data files and created the spectra, the detector response matrix, and the effective area using XMM SAS version 7.1. In the MOS2 image the cluster centre unfortunately lies on a gap between two CCDs. Therefore we excluded this camera from our analysis. We varied the aperture of the source extraction region and found that with a radius of $45^{\prime \prime}$ the cluster redshift and temperature are determined with smallest uncertainties.

We used XSPEC version 12.0 to subtract the background spectra and to fit MEKAL plasma models. The MEKAL code (Kaastra 1992; Liedahl 1995) allows to set a metal abundance parameter relative to the solar values provided by Anders \& Grevesse (1989). Fits were carried out using the Cash-statistics and a binning with at least one photon per spectral bin. The Galactic neutral hydrogen column density in the direction of 2XMM 0830 is $N_{\mathrm{H}}=4.0 \times 10^{20} \mathrm{~cm}^{-2}$, as obtained from radio data (Dickey \& Lockman 1990). The Fe K-line is visible in both spectra at $k_{\mathrm{B}} T \sim 3 \mathrm{keV}$, see Fig. 2. Hence, the redshift

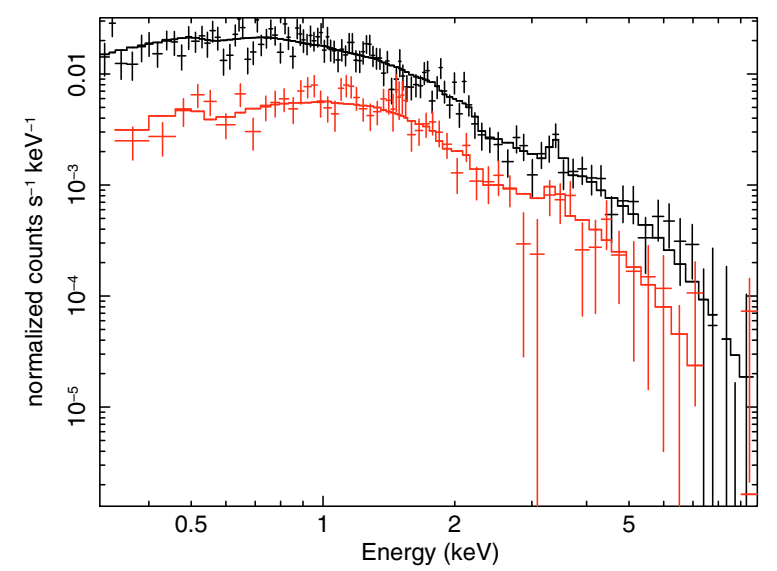

Fig. 2. EPIC PN (upper spectrum in black) and MOS1 (lower spectrum in red) data with best fitting MEKAL model.

of 2XMM 0830 is unambiguously determined by the X-ray data, see Fig. 3. The best-fit redshift, temperature, and metallicity are $z=0.99 \pm 0.03, k_{\mathrm{B}} T=8.2 \pm 0.9 \mathrm{keV}$, and $Z / Z_{\odot}=0.32 \pm$ 0.18 . The latter is in agreement with the results of Balestra et al. (2007) and Maughan et al. (2008), who analysed the Chandra spectra of larger cluster samples and found $\left\langle Z \mid Z_{\odot}\right\rangle=0.28$ and $\left\langle Z \mid Z_{\odot}\right\rangle=0.2$ as the mean abundances at $z \sim 1$.

The spectral fit provides also the background subtracted bolometric X-ray flux $F_{\text {bol }}=2.4 \times 10^{-13} \mathrm{erg} \mathrm{s}^{-1} \mathrm{~cm}^{-2}$ within the aperture of $45^{\prime \prime}$. Using the concordance cosmological model allows to calculate the intrinsic luminosity of the cluster. However, the used aperture corresponds to a radius significantly smaller than $r_{500}$. We estimate the missing flux by approximating a $\beta$-model. Figure 4 shows the spherically averaged surface photon density for the MOS1 image. Allowing all parameters to vary in a least-square fit we find for the slope $\beta=0.73 \pm 0.1$ and for the core radius $r_{\mathrm{c}}=(24 \pm 4)^{\prime \prime}$. Now, we determine $r_{500}$ by the temperature-radius relation given in O'Hara et al. (2007). For the redshift and temperature of 2XMM 0830 derived above we obtain $r_{500}=(860 \pm 50) \mathrm{kpc}$, corresponding to $(107 \pm 6)^{\prime \prime}$. With the results of the $\beta$-profile fit we find for the intrinsic luminosity of 2 XMM $0830 L_{\text {bol }}\left(<r_{500}\right)=1.8 \times 10^{45} \mathrm{erg} \mathrm{s}^{-1}$.

The mass of a cluster can be estimated by a model based on spherical symmetry and hydrostatic equilibrium. Since for 2XMM 0830 the determination of the temperature profile is not possible, we additionally assume isothermality. As a result, the total mass is given by

$M_{500} \sim \frac{3 \beta}{G} \frac{k_{\mathrm{B}} T r_{500}}{\mu m_{\mathrm{p}}} \frac{\left(r / r_{\mathrm{c}}\right)^{2}}{1+\left(r / r_{\mathrm{c}}\right)^{2}}$

(see e.g. Hicks et al. 2007).

Using the properties of $2 \mathrm{XMM} 0830$ as derived above we obtain for the mass $M_{500}=(5.6 \pm 1.0) \times 10^{14} M_{\odot}$, where we set $\mu=0.6$ as expected for a fully ionised ICM. For comparison, the mass-temperature relation of Vikhlinin et al. (2006) predicts $(3.8 \pm 0.7) \times 10^{14} M_{\odot}$, which is within $2 \sigma$ of our derived value.

The luminosity of this cluster agrees within the errors with the local $L-T$ relation (Markevitch 1998). However, Kotov \& Vikhlinin (1998) and Maughan et al. (2006) argued that the luminosity scales with redshift roughly in concordance with the expectations of self-similar cluster evolution. Contrarily, O'Hara et al. (2007) found only very little evolution of the $L_{500}-T$-relation in a recent analysis of a sample of 70 clusters observed with Chandra. A similar discrepancy has been found for the most distant cluster, XMMXCS J2215.9-1738; it 

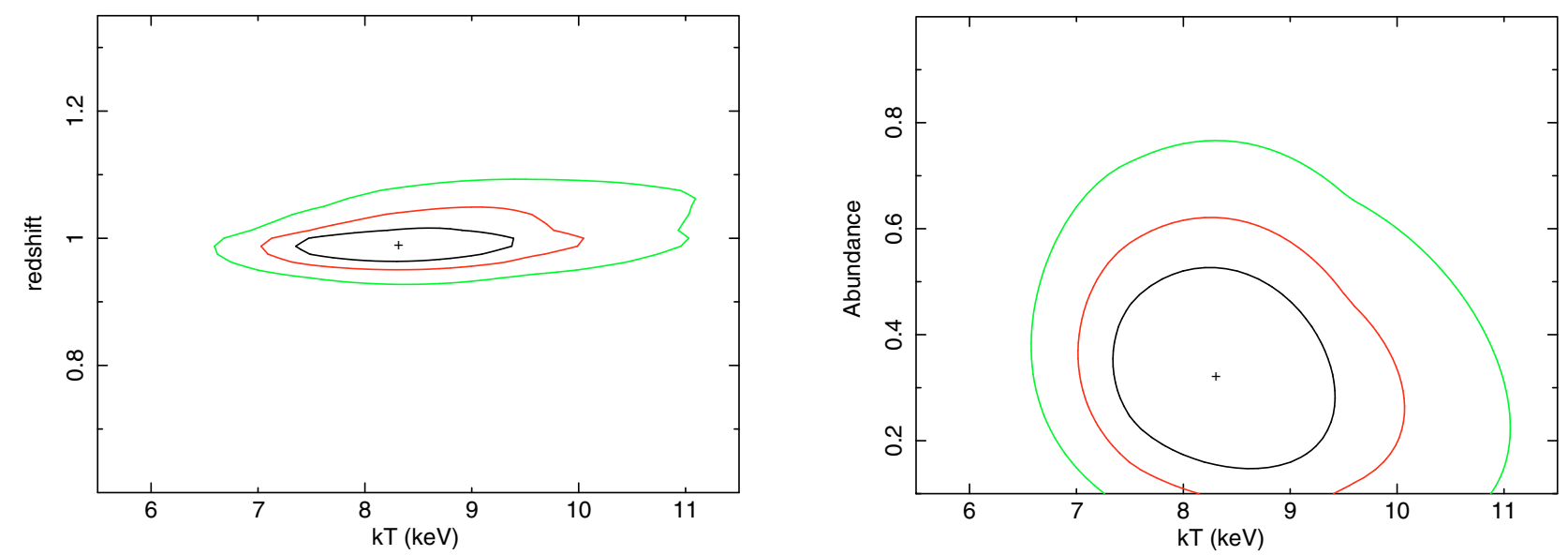

Fig. 3. Left: confidence contours for redshift and temperature determined from the X-ray spectra. Starting from the innermost contour, the levels indicate $68 \%, 90 \%$, and $99 \%$ confidence. Right: same confidence contours for metal abundance and temperature.

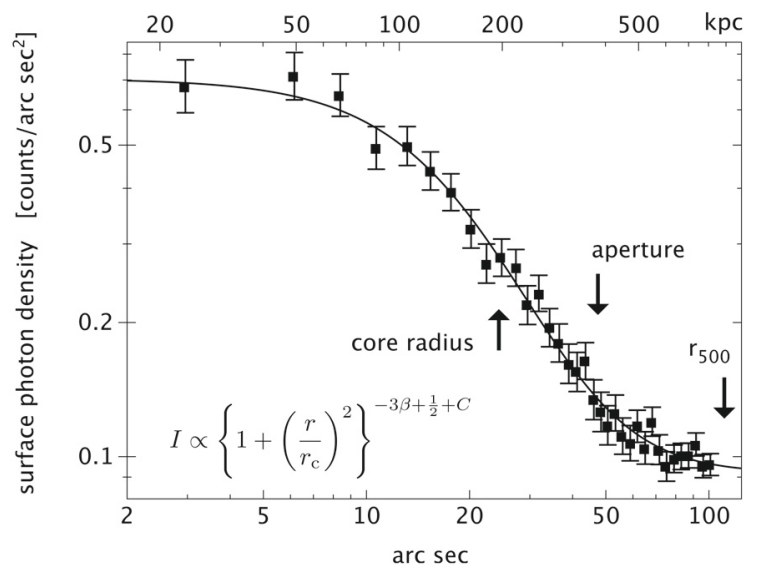

Fig. 4. Spherically averaged surface luminosity profile used to extrapolate the X-ray luminosity to $r_{500}$.

is under-luminous even compared to the local $L_{500}-T$-relation (Hilton et al. 2007). This illustrates that the evolution of gas scaling properties is still poorly constrained. Hence, a larger high redshift cluster sample is needed and selection effects have to be modelled properly.

\section{Optical imaging}

No cluster galaxies or any other plausible optical counterparts for an extended X-ray source are visible in SDSS images at the position of 2XMM0830 (Fig. 5). A rather blue galaxy (SDSS J083026.39+524130.5, $r-z=0.63$ ) with disk-like appearance some 6 arcsec south of the X-ray position is most likely a foreground spiral galaxy.

We have obtained deep $r_{\text {sloan }}$ and $z_{\text {sloan }}$ images in the field of 2XMM 0830 using the LBC cameras of the LBT. The $r$-band image was taken with the blue LBC camera and the $z$-band image with the red camera. Both bands have total exposure times of $18 \mathrm{~min}(9 \times 2 \mathrm{~min})$ and were taken on May 10th, 2008. The data reduction was accomplished with a pipeline software based on the Garching-Bonn Deep Survey (GaBoDS) pipeline, which has been modified at AIP to process the LBC data. The astrometry and photometry of the stacked images has been tied to the SDSS. The image quality of the reduced images is $F W H M=1.1 \mathrm{arcsec}$ in the $z$-band image and 1.5 arcsec in the $r$-band. A colour composite of the two images is shown in Fig. 6. The LBT images

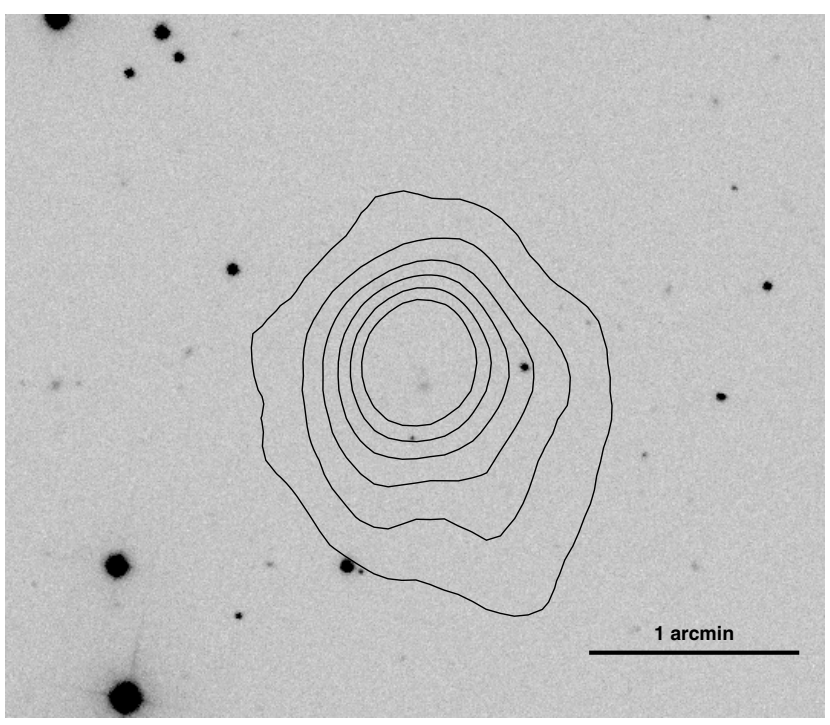

Fig. 5. SDSS $i$-band image with EPIC MOS1 X-ray contours of 2XMM 0830 overlaid. The object 6 arcsec south south of the X-ray position is SDSS J083026.39+524130.5.

reveal a clear overdensity of red galaxies within the X-ray contours. The brightest cluster galaxy is located close to the peak of the X-ray emission. Its $z$-band magnitude of 19.44 and colour $r-z=2.15$ are consistent with the X-ray redshift $z=1$. A detailed analysis of the LBT data will be presented in a forthcoming paper.

\section{Summary}

Based on the X-ray data we show that 2XMM J083026.2+ 524133 is a massive cluster at $z \sim 1$. Its luminosity of $1.8 \times$ $10^{45} \mathrm{erg} \mathrm{s}^{-1}$ and temperature of $8.2 \pm 0.8 \mathrm{keV}$ are unsurpassed at its distance and beyond. The only comparable known cluster is CL J1226.9+3332 (Ebeling et al. 2001) which is less distant at $z=0.89$, but even more luminous and hotter $\left(L_{\text {bol }}=5 \times\right.$ $10^{45} \mathrm{erg} \mathrm{s}^{-1}, k T=10.4 \mathrm{keV}$, Maughan et al. 2007).

The luminosity of 2XMM0830 is possibly enhanced by a cool core. Since its X-ray flux makes 2XMM 0830 an ideal target for more detailed investigations in X-rays, we have applied for a deep Chandra observation in order to investigate a possible cool core of the cluster and to establish an improved mass model. 


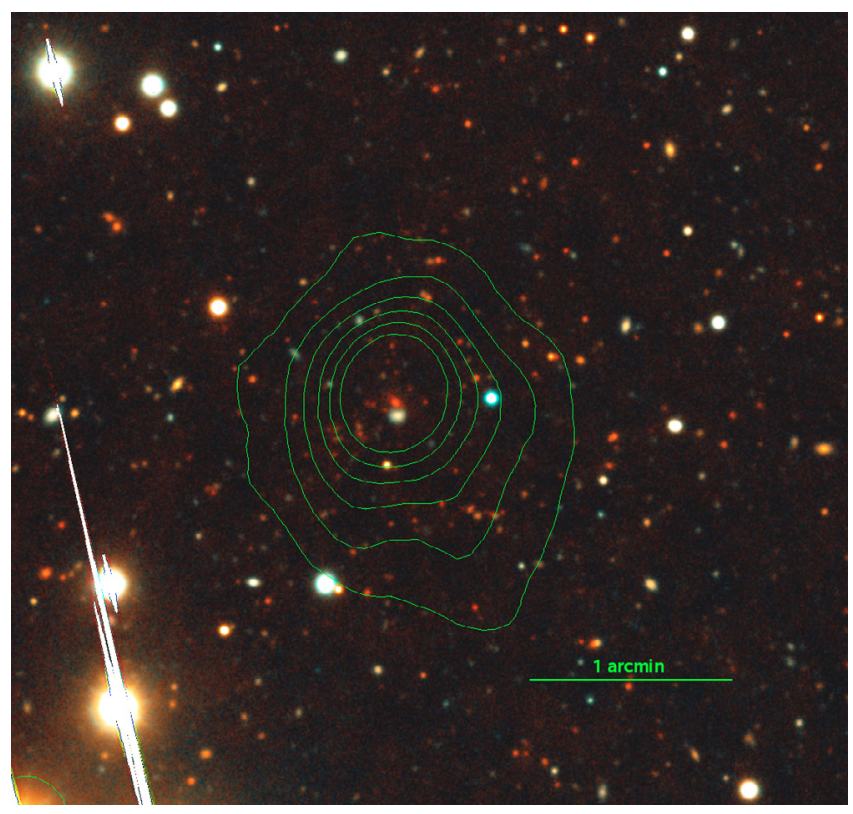

Fig. 6. LBT $r$ - and $z$-band colour composite image with XMM EPIC contours (green). The blue galaxy just south of the X-ray centre is probably unrelated to the cluster and corresponds to SDSS J083026.39+524130.5.

How likely is it to find a cluster with the mass of 2XMM 0830 in the XMM-Newton serendipitous survey data? We have estimated the number of clusters with $M \geq 5.6 \times$ $10^{14} M_{\odot}$ expected in the redshift shell $0.9<z<1.1$ and $0.3 \%$ of the sky corresponding to our survey area in the 2XMM-SDSS overlap. The mass function of Gottlöber et al. (2006), based on the large MareNostrum Universe SPH simulation using the "WMAP first year" cosmology $\left(\sigma_{8}=0.9\right)$ predicts 5 clusters. Calculating the mass function following Press \& Schechter (1974) for the "WMAP 3 years" cosmological parameters with $\sigma_{8}=0.76$ predicts 0.1 clusters in our survey. Changing $\sigma_{8}$ to 0.82 increases the expected cluster count to 0.4 . Given the uncertainty of our mass estimate, we conclude that the XMM-Newton discovery of one or few clusters like 2XMM 0830 is within the expectations. However, we will have to await the eROSITA allsky survey (Predehl et al. 2006) to find a larger number of these very massive clusters.

Acknowledgements. G.L. and M.H. acknowledge support by the Deutsches Zentrum für Luft- und Raumfahrt (DLR) under contracts 50 QR 0802 and 50 OX 0201. J.K. is supported by the DFG priority programme SPP1177 (grant No. SCHW563/23-1). This work is based on observations obtained with XMM-Newton and on data acquired using the Large Binocular Telescope (LBT). We thank Alexander Knebe for providing code to calculate the Press-Schechter mass function.

\section{References}

Anders, E., \& Grevesse, N. 1989, Geochim. Cosmochim. Acta, 53, 197 Balestra, I., Tozzi, P., Ettori, S., et al. 2007, A\&A, 462, 429 Borgani, S., \& Guzzo, L. 2001, Nature, 409, 39 Dickey, J. M., \& Lockman, F. J. 1990, ARA\&A, 28, 215 Ebeling, H., Jones, L. R., Fairley, B. W., et al. 2001, ApJ, 548, L23 Finoguenov, A., Guzzo, L., Hasinger, G., et al. 2007, ApJS, 172, 254 Gottlöber, S., Yepes, G., Khalatyan, A., Sevilla, R., \& Turchaninov, V. 2006, in The dark side of the Universe, DSU 2006, AIP Conf. Proc., 878, 3 Henry, J. P., Gioia, I. M., Maccacaro, T., et al. 1992, ApJ, 386, 408 Hicks, A. K., Ellingson, E., Hoekstra, H., et al. 2007 [arXiv : 0708. 3671] Hilton, M., Collins, C. A., Stanford, S. A., et al. 2007, ApJ, 670, 1000 Kaastra, J. S. 1992, Internal SRON-Leiden Report, updated version 2.0 Kotov, O., \& Vikhlinin, A. 2005, ApJ, 633, 781

Liedahl, D. A., Osterheld, A. L., \& Goldstein, W. H. 1995, ApJ, 438, L115 Markevitch, M. 1998, ApJ, 504, 27

Maughan, B. J., Jones, L. R., Ebeling, H., \& Scharf, C. 2006, MNRAS, 365, 509 Maughan, B. J., Jones, C., Jones, L. R., \& Van Speybroeck, L. 2007, ApJ, 659, 1125

Maughan, B. J., Jones, L. R., Forman, H., \& Van Speyboeck, L. 2008, ApJS, 174,117

Mullis, C. R., Rosati, P., Lamer, G., et al. 2005, ApJ, 623, L85

O'Hara, T. B., Mohr, J. J., \& Sanderson, A. J. R. 2007 [arXiv: 0710. 5782]

Pierre, M., Pacaud, F., Duc, P. A., et al. 2006, MNRAS, 372, 591

Predehl, P., Hasinger, G., Böhringer, H., et al. 2006, Proc. SPIE, 6266, 19

Press, W. H., \& Schechter, P. 1974, ApJ, 187, 425

Romer, K. A., Viana, P. T. P., Liddle, A. R., \& Mann, R. G. 2001, ApJ, 547, 594 Rosati, P., Borgani, S., \& Norman, C. 2002, ARA\&A, 40, 539

Poole, G. B., Babul, A., McCarthy, I. G., Sanderson, A. J. R., \& Fardal, M. A. 2008 [arXiv: 0804.1552]

Stanford, S. A., Romer, K. A., Sabirli, K., et al. 2006, ApJ, 646, L13

Vikhlinin, A., Kravtsov, A., Forman, W., et al. 2006, ApJ, 640, 691

Watson, M. G., et al. 2008, A\&A, in preparation 